\title{
Factores de riesgo asociados a recidiva de carcinoma papilar de tiroides
}

\author{
Risk factors associated with recurrence of papillary thyroid carcinoma
}

\author{
Víctor Hernández A', Maricela Jiménez-López¹, Salvador Serrano F¹, Gabriela Obregón G², \\ Claudia Itzá Pérez M².
}

\begin{abstract}
RESUMEN
Introducción: Aunque el carcinoma papilar de tiroides (CPT) tiene una buena sobrevida, en el $30 \%$ de los casos recidivará a largo plazo. Se han descrito factores pronósticos como el tamaño, histopatología, procedimiento quirúrgico y administración de yodo radiactivo.

Objetivo: Este trabajo pretende determinar factores de riesgo de recidiva a largo plazo.

Material y método: Se realizó un estudio retrospectivo y observacional, se incluyeron a los pacientes sometidos a cirugía por CPT con seguimiento a 10 años, y se analizaron variables clínicas y bioquímicas relacionadas con la recidiva a largo plazo.

Resultados: Se identificaron 91 pacientes con seguimiento de 10 años. No se encontró relación para recidiva con historia familiar oncológica, enfermedad tiroidea previa, pero sí con tabaquismo (p 0,040). Se encontraron a 27 (29\%) con recidiva, en relación a lesiones $>3 \mathrm{~cm}(p 0,05)$, y CPT multicéntrico $(p 0,003)$.

Conclusión: El tiempo de evolución prolongado favorece el crecimiento de las lesiones, y la diseminación de la enfermedad, así como la recidiva. EI CPT es una enfermedad con capacidad metastásica a largo plazo, que requiere un seguimiento cercano y detección oportuna de pacientes susceptibles de recidiva. El tiempo entre el diagnóstico y la cirugía es un factor fundamental para el crecimiento de las lesiones y la propagación de la enfermedad, por lo que se debe reducir el tiempo de espera, evitando así las lesiones de mayor tamaño, diseminación de células tumorales y la recidiva con peor pronóstico para los pacientes.
\end{abstract}

Palabras clave: Cáncer papilar de tiroides; tiroidectomía; recidiva; cirugía endocrina.

\footnotetext{
ABSTRACT

Introduction: The papillary thyroid cancer has good survival rate, however, 30\% of the patients will have a recurrence. Prognostic factors have been described such as size, histopathology, surgical procedure and administration of radioactive iodine.

1 Servicio de Gastrocirugía, Hospital de Especialidades, Centro Médico Nacional Siglo XXI, Instituto Mexicano del Seguro Social (IMSS), México.

2 Servicio de Cirugía de Cabeza y Cuello. Hospital de Especialidades, Centro Médico Nacional Siglo XXI, Instituto Mexicano del Seguro Social (IMSS), México.

*Los autores declaran no tener conflictos de interés.

Recibido el 6 de junio, 2018. Aceptado el 2 de septiembre, 2018.
} 
Aim: To determine preventable risk factors for long-term recurrence.

Material and method: This is a retrospective and observational study, patients undergoing surgery for CPT and 10 year follow up were included to analyze clinical and biochemical variables related to long-term recurrence.

Results: Ninety-one patients with a 10-year follow-up were identified. No relationship was found for recurrence with oncological family history, previous thyroid disease, but smoking was a risk factor ( $p$ 0.040). We found 27 (29\%) with relapse, in relation to lesions $>3 \mathrm{~cm}(p$ 0.05), and multicentric PTC (p 0.003).

Conclusion: The long evolution time favors the growth of lesions, the spread of the disease, as well as the recurrence. The CPT is a disease with long-term metastatic capacity; it requires close monitoring and opportune detection of patients susceptible to recurrence. The time between diagnosis and surgery is a fundamental factor for the growth of the lesions and the spread of the disease, so the waiting time must be reduced, thus avoiding larger lesions, malignant cell dissemination and recurrence with worse prognosis for patients.

Key words: Papillary thyroid cancer; thyroidectomy; recurrence; endocrine surgery.

\section{INTRODUCCIÓN}

El carcinoma papilar de tiroides (CPT) es una entidad frecuente, y usualmente se acompaña de un curso favorable, con una sobrevida de hasta el $90 \%$ a 10 años ${ }^{1,2}$. Sin embargo, este $10 \%$ restante es susceptible de desarrollar recidiva local, enfermedad diseminada ${ }^{3}$, o incluso de morir por esta enfermedad ${ }^{2,4-6}$. La incidencia del CPT se ha incrementado en la última década en todo el mundo, probablemente debido a la implementación sistemática de técnicas de imagen que permiten un mejor diagnóstico ${ }^{7-9}$ e identificación oportuna de lesiones pequeñas, y de enfermedad a distancia, ya que las metástasis a ganglios linfáticos ocurren hasta en el $80 \%$ de los pacientes ${ }^{7,8,10}$.

Se han descrito en la literatura varios factores pronósticos como edad de presentación, sexo, tamaño y extensión del tumor primario, presencia de metástasis, características histopatológicas, procedimiento quirúrgico inicial y administración de yodo radiactivo posoperatorio ${ }^{11}$. En las últimas décadas se han desarrollado escalas de evaluación para predecir la recidiva y mortalidad de esta enfermedad, algunos ejemplos con mayor difusión son MACIS (metástasis, edad, cirugía, invasión local y tamaño del tumor $)^{7,11}$ y el sistema TNM. Estas escalas de factores de riesgo se basan fundamentalmente en la biología de este tipo de tumor, incluyendo su capacidad para crecer, invadir y diseminarse $\mathrm{e}^{7,10,12}$.
La recidiva del CPT puede presentarse de 3 formas principales: enfermedad a distancia, recidiva local (en tejidos blandos) y en nódulos linfáticos ${ }^{7,13}$, las primeras dos formas evidencian la biología agresiva de los tumores, y aunque son infrecuentes, se presentan una mayor tasa de mortalidad para estos pacientes. La forma más frecuente, y la más estudiada, es la recidiva en ganglios linfáticos cervicales que ocurre de forma temprana, usualmente localizada en el nivel central (C-VI), que por su pequeño tamaño puede escaparse de la exploración quirúrgica y que pueden aparecer en los resultados de patología en el $60 \%$ de los pacientes ${ }^{14,15}$.

La cirugía en estos pacientes, deberá entonces tener como metas principales, ser adecuada para remover el tumor y la enfermedad diseminada a tejidos locales y a nódulos linfáticos, ya que es un factor determinante para la recidiva y la persistencia de enfermedad y es de las pocas variables controlables para evitar la recidiva que podría influenciar el pronóstico a largo plazo ${ }^{15}$.

La incidencia de recidiva y el tipo de cirugía realizada se han visto también influenciadas por el uso de yodo radioactivo como terapia adyuvante en el posoperatorio que quizá ha hecho que la disección de nódulos no palpables no sea esencial ${ }^{14,15}$, sin embargo, es importante la identificación de pacientes en riesgo de recidiva para su manejo y seguimiento adecuados. 


\section{OBJETIVO}

Este trabajo tiene por objetivo identificar los factores de riesgo más relevantes en esta población de pacientes con CPT con la finalidad de establecer un precedente de factores de riesgo prevenibles que al modificarse mejoren los resultados posoperatorios en términos de tiempo libre de enfermedad a largo plazo para estos pacientes.

\section{MATERIAL Y MÉTODO}

Se realizó un estudio observacional y retrospectivo con una fase de reclutamiento de pacientes durante el periodo de enero de 1998 a diciembre de 2009. Se tomaron como criterios de inclusión: pacientes mayores de 18 años, usuarios de la sanidad pública en el Instituto Mexicano del Seguro Social (IMSS), que fueron enviados al centro por sospecha de CPT y se sometieron a cirugía como tratamiento. Se excluyeron pacientes que habían sido tratados o intervenidos previamente por diagnóstico de $\mathrm{CPT}$, aquellos que después de la cirugía se hiciera diagnóstico histopatológico de una neoplasia distinta y los que se perdieron en el seguimiento.

Se recabaron las historias clínicas para obtener datos demográficos y antecedentes médicos de los pacientes; se revisaron las pruebas de imagen con ecografía realizada previo al tratamiento para registrar las características preoperatorias de la lesión; y se revisaron los reportes de patología de piezas quirúrgicas para confirmar el diagnóstico de CPT en la cirugía inicial y de recidiva en las biopsias 0 reintervenciones posteriores. En este estudio se definió recidiva a la presencia de CPT en la pieza quirúrgica de la biopsia o reintervención confirmada por el servicio de anatomía patológica.

\section{Análisis estadístico}

Se realizó el análisis estadístico con el paquete de software SPSS v.23 con medidas de tendencia central, comparación de medias con la prueba de $T$ de Student para las variables cuantitativas y estadísticos descriptivos con la prueba de $x^{2}$ para las variables categóricas. Se consideraron valores significativos para estas variables a un valor de $p$ $<0,05$ y se consideró una tendencia estadística de relación a valores entre 0,05 y 1 . Posteriormente se analizaron las variables significativas asociadas con recidiva de forma individual y en conjunto con una prueba de regresión logística multivariada para obtener el impacto y fuerza estadística de cada variable. De forma paralela, se analizaron a las variables demográficas, características por ecografía de las lesiones y características quirúrgicas con la supervivencia libre de enfermedad usando curvas de Kaplan Meier para demostrar el impacto de éstas en los eventos de recidiva a lo largo del seguimiento.

\section{RESULTADOS}

\section{Descripción de la serie}

Se identificaron 91 pacientes en el periodo entre enero de 1998 a diciembre de 2009 con un seguimiento con una media de 10 años. De estos pacientes $79(86 \%)$ fueron mujeres y $12(13,2 \%)$ hombres, con una media de edad de 47 años (DE \pm 15). Se encontraron como antecedentes relevantes, historia familiar oncológica en 21 (23\%) pacientes, $11(12 \%)$ con antecedente de diabetes mellitus tipo 2, y 31 (34\%) pacientes con historia de enfermedad tiroidea. En cuanto a factores de riesgo para neoplasia en este grupo, se encontraron 2 casos con exposición a radiación y 21 (23\%) con antecedente de tabaquismo.

En el cuadro clínico, se encontró la lesión como un hallazgo incidental en individuos asintomáticos en $26(29 \%)$ pacientes. En el resto, se presentó disfonía en $16(18 \%)$, disfagia en $23(25 \%)$, disnea en $11(12 \%)$, nódulo palpable en $49(53 \%)$, y parálisis de las cuerdas vocales en $3(3,3 \%)$. En la exploración física se encontró un nódulo único en $66(72 \%)$, móvil en $46(50 \%)$, consistencia pétrea en 36 (39\%), y adenomegalias en 23 (25\%). Se encontró un valor de TSH medio al diagnóstico de $3,1 \mathrm{mU} / \mathrm{l}(\mathrm{DE} \pm 8,5)$ y $\mathrm{T} 4$ libre de $2,5 \mathrm{mU} / \mathrm{l}(\mathrm{DE} \pm$ 11). Al interrogatorio, los pacientes refirieron una media del tiempo de evolución desde el inicio de sus síntomas hasta que acudieron al médico de 29 meses (DE \pm 36$)$. En 17 (18\%) de los casos, los pacientes notaron un crecimiento rápido de la lesión en el mes previo a la consulta que los motivó a buscar atención médica. 
En el estudio preoperatorio de los pacientes, se solicitó USG a 56 (61\%), en el cual se observó un tamaño de la lesión con una media de 4,3 (DE \pm 3.3 ), una lesión de consistencia sólida en 18 $(32 \%)$, quística $4(7 \%)$ y mixta en $19(33 \%)$. Las lesiones identificadas presentaron bordes indefinidos en 51 (92\%), vasculatura en $10(17 \%)$, microcalcificaciones en $17(30 \%)$ y presencia de adenopatías cervicales en 18 (32\%). Se solicitó biopsia preoperatoria de la lesión en 76 pacientes de los cuales se encontró carcinoma papilar de tiroides (CPT) en 43 (55\%).

El tiempo de espera para la programación de cirugía después de la primera consulta fue de 132 días debido a la necesidad de completar el protocolo preoperatorio y el volumen de pacientes manejado por el centro hospitalario. El tipo de cirugía más frecuente fue la tiroidectomía total en $83(91 \%)$ de los casos; con disección de nivel VI en $79(86 \%)$ y disección selectiva de cuello (DSC) en $33(36 \%)$.

En el análisis histopatológico de las piezas quirúrgicas, el tamaño medio del diámetro mayor es de $3,3 \mathrm{~cm}(\mathrm{DE} \pm 2.6)$; se reportó CPT clásico en 59 (64\%), patrón folicular 14 (15.4\%), multicéntrico en $4(4.4 \%)$, y carcinoma medular de tiroides en $2(2,2 \%$; éstos se excluyeron ya que presenta un comportamiento biológico distinto). Se observó invasión multifocal en $27(29,7 \%)$, invasión a la cápsula en $51(56 \%)$, permeación linfática en 17 $(18,7 \%)$, permeación vascular en $26(28,6 \%)$, y ruptura capsular en $8(8,8 \%)$. El número de ganglios resecados positivos para malignidad por paciente fue una media de 2,4.

\section{Descripción de los pacientes con recidiva de CPT}

La sospecha de recidiva se determinó por la presencia de tiroglobulina elevada en estado de supresión de TSH, combinado con adenopatías en ultrasonido cervical. Se encontraron a $40(44 \%)$ pacientes con sospecha de recidiva en los que se realizó reintervención con una media de 357 días transcurridos entre la primera y la segunda cirugía. En la reintervención, la cirugía realizada con mayor frecuencia fue la DSC en $26(65 \%)$ pacientes y exploración de cuello en 7 (17\%). Después de la reintervención se encontraron 27 (67\%) pacientes con reporte histopatológico de recidiva de CPT. Las características de estos pacientes se resumen en la Tabla 1.

Durante el seguimiento posoperatorio se realizaron mediciones de perfil tiroideo con tirogIobulina con una media de $23 \mathrm{ng} / \mathrm{dl}(\mathrm{DE} \pm 83)$ y $\mathrm{TSH}$ de $47 \mathrm{mU} / \mathrm{l}(\mathrm{DE} \pm 222)$, sin embargo, no se encontraron asociaciones significativas en estos valores para riesgo de recidiva. En esta población se dio tratamiento posoperatorio con ${ }^{131}$ en 79 $(86 \%)$ pacientes, pero no se encontró relación con la recidiva comparado con aquellos que no la recibieron.

\section{Análisis estadístico de las variables de riesgo}

Se realizó una prueba de T de Student para las variables cuantitativas, encontrando relación con un mayor tamaño de la lesión en la exploración física inicial $(p=0,026)$, y el mayor tiempo transcurrido entre la primera consulta y la cirugía ( $p=0,042)$, y entre ésta y la reintervención por sospecha de recidiva $(p=0,004)$. Para las variables categóricas se realizó una $X^{2}$ encontrando valores estadísticamente significativos únicamente para el tabaquismo $(p=0,040)$ como factor de riego para recidiva. De las variables clínicas, de exploración física y quirúrgicas, se realizó una regresión logística multivariada con aquellas que fueron estadísticamente significativas relacionadas con recidiva para evaluar el impacto de cada variable para este evento (Tabla 1).

\section{Supervivencia libre de recidiva de la serie}

Se realizaron curvas de Kaplan Meier para evaluar los factores que pueden asociarse a eventos de recidiva encontrando una relación relevante para nódulo único ( $p=0,034)$, y móvil $(p=0,033)$ a la exploración física, y una tendencia estadística para las lesiones de rápido crecimiento $(0,073)$. Otros signos y síntomas como disfonía $(p=0,550)$, disfagia ( $p=0,171)$, disnea $(0,858)$, consistencia del nódulo palpable $(p=0,407)$, y presencia de adenomegalias $(p=0,138)$ no resultaron significativas en esta prueba (Figura 1).

Entre las variables transquirúrgicas, se encontró una tendencia en la asociación con el tamaño de la lesión $>3 \mathrm{~cm}(p=0,054)$, y diferencia significativa con el resultado del reporte histopatológico, 
Tabla 1. Variables evaluadas para los pacientes con recidiva del carcinoma papilar de tiroides ( $N=27$ )

\begin{tabular}{|c|c|c|c|}
\hline Variable & $n(\%)$ & Valor de $p$ & Impacto (HR) \\
\hline Sexo (femenino) & $24(88 \%)$ & 0,704 & \\
\hline Edad $>45$ años & $11(40 \%)$ & 0,419 & \\
\hline Historia familiar oncológica & $4(14 \%)$ & 0,104 & \\
\hline Diabetes mellitus 2 & $2(7 \%)$ & 0,374 & \\
\hline Enfermedad tiroidea previa & $11(40 \%)$ & 0,383 & \\
\hline Exposición a radiación & 0 & & \\
\hline Tabaquismo & $10(37 \%)$ & 0,040 & 2,80 \\
\hline \multicolumn{4}{|l|}{ Exploración física } \\
\hline Tamaño de lesión mayor a $>3 \mathrm{~cm}$ & $13(48 \%)$ & 0,026 & 1,56 \\
\hline Disfonía & $7(25 \%)$ & 0,180 & \\
\hline Disfagia & $8(29 \%)$ & 0,536 & \\
\hline Disnea & $3(11 \%)$ & 0,853 & \\
\hline Nódulo único & $21(77 \%)$ & 0,466 & \\
\hline Nódulo móvil & $15(55 \%)$ & 0,535 & \\
\hline Consistencia pétrea & $8(29 \%)$ & 0,208 & \\
\hline Adenomegalias cervicales & $8(29 \%)$ & 0,674 & \\
\hline \multicolumn{4}{|l|}{ Características de la ecografía } \\
\hline Bordes no definidos & $26(96 \%)$ & 0,354 & \\
\hline Lesión vascularizada & $2(7 \%)$ & 0,478 & \\
\hline Microcalcificaciones & $2(7 \%)$ & 0,146 & \\
\hline \multicolumn{4}{|l|}{ Características de la Cirugía } \\
\hline Tiempo de espera de la $1^{\text {a }}$ cirugía (media, DE) & $132( \pm 285)$ & 0,042 & 0,99 \\
\hline Invasión a la cápsula & $18(66 \%)$ & 0,185 & \\
\hline Permeación linfática & $6(22 \%)$ & 0,574 & \\
\hline Permeación vascular & $8(29 \%)$ & 0,885 & \\
\hline
\end{tabular}

siendo de peor pronóstico el CPT multicéntrico ( $p$ $=0,003$ ) (Figura 2). En las variables del posoperatorio evaluadas se reportó hematoma en $4(4,3 \%)$ de los pacientes, lesión al nervio laríngeo recurrente en $5(5,5 \%)$, seroma en $2(2,2 \%)$, hipoparatiroidismo temporal en $60(66 \%)$ y permanente en 22 (24\%), sin embargo ninguno se asoció a riesgo de recidiva.

\section{DISCUSIÓN}

En este trabajo, se estudió a una serie de pacientes con un seguimiento prolongado posterior a la intervención por diagnóstico de CPT, se ha podido demostrar que entre los factores preoperatorios más importantes y modificables, uno de los factores de relevancia, además del tiempo de diagnóstico y tratamiento, es el tabaquismo por el aumento de casi el doble del riesgo para la recidiva (HR 2,8), sin ser de importancia estadística el índice tabáquico de cada paciente, lo cual pueda deberse a la capacidad mutagénica de las sustancias contenidas en el cigarrillo y que es prevenible modificando hábitos de vida que podrían tener un importante impacto sobre la recidiva en este grupo de pacientes.

Además, es importante mencionar que las lesiones mayores, en este caso con más de 3 $\mathrm{cm}$ se asocian a un incremento en el riesgo de recidiva (HR 1,53) como ya se ha demostrado en estudios previos ${ }^{7}$, sin embargo, en este estudio se le suma la importancia del tiempo prolongado de evolución ya sea por retraso en la búsqueda de atención médica o por el tiempo en lista de espera para cirugía. Es muy importante en estos casos que se tenga en cuenta que el mayor tiempo de latencia de la enfermedad, favorecerá 


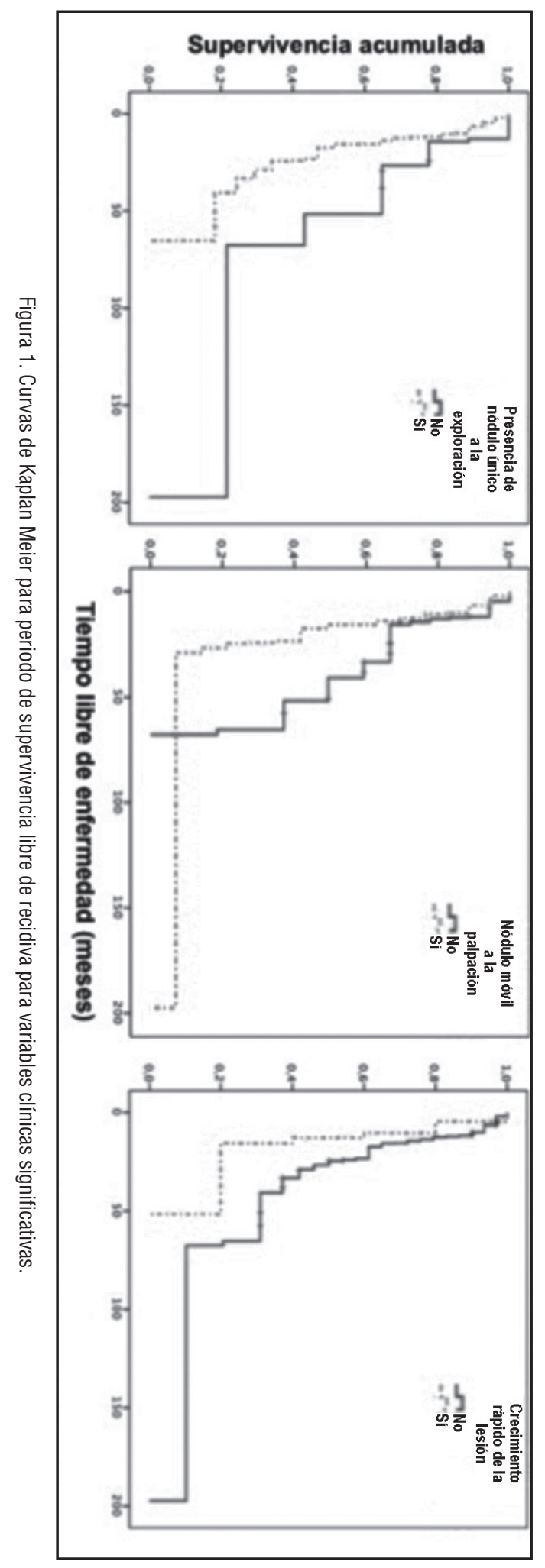




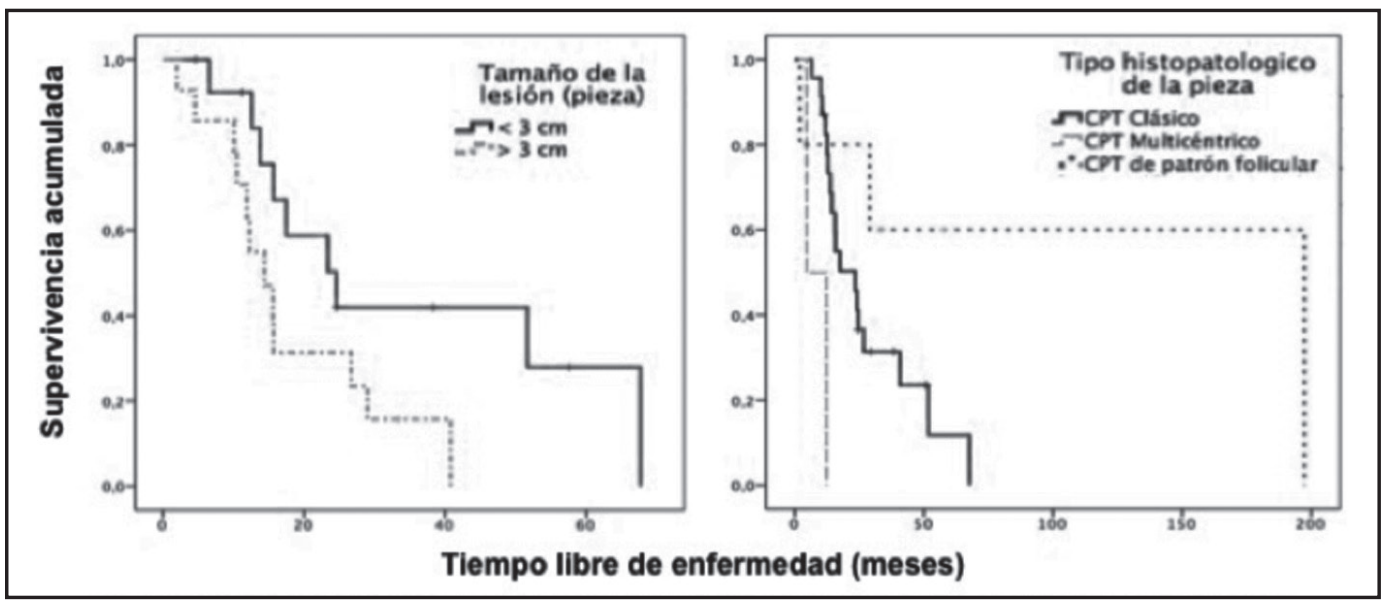

Figura 2. Curvas de Kaplan Meier para variables quirúrgicas que resultaron significativas.

el crecimiento de la lesión neoplásica, su progresión hacia tejidos blandos contiguos y la diseminación de la enfermedad por vía linfática o hematógena que favorezca eventos de recidiva posteriores ya que se permite la salida de células circulantes tumorales que permanezcan en el paciente aun después de recibir el tratamiento adecuado.

En la predicción del riesgo de recidiva y mortalidad, sugerimos que se deberá tomar en cuenta la biología del tumor y las características del paciente, al construir una escala de factores de riesgo para CPT en este grupo de pacientes consideramos de gran importancia incluir el tiempo de evolución, el tamaño del tumor, su tipo histológico (en este caso multicéntrico como de peor pronóstico), y su agresividad; variables que han mostrado su importancia en múltiples análisis multivariados en la literatura ${ }^{7,13,14}$ y con lo cual coinciden nuestros resultados.

\section{CONCLUSIÓN}

En este trabajo, el propósito de la evaluación de las variables que influyen en la recidiva del CPT es identificar los cambios que pueden realizarse para estos pacientes en el abordaje preoperatorio, transoperatorio y seguimiento para obtener mejores resultados a largo plazo y construir un sistema para valoración que mejore la práctica clínica diaria. Como se ha comentado previamente, el CPT es una enfermedad con capacidad metastásica a largo plazo, que requiere un seguimiento cercano y la detección oportuna de los pacientes susceptibles de recidiva.

Los factores de riesgo identificados en este trabajo son en gran medida modificables ya que la mayoría son características del paciente o del sistema de valoración. La suspensión del hábito tabáquico puede reducir el riesgo y mejorar los resultados posoperatorios; y el tiempo de espera para la intervención quirúrgica es un factor fundamental para el crecimiento de las lesiones y la propagación de la enfermedad, por lo que se debe reducir en la práctica clínica habitual el tiempo en protocolos de evaluación que provoquen este retraso de la cirugía y evitando así las lesiones de mayor tamaño y la diseminación de células tumorales que provoquen enfermedad sistémica y el mayor riesgo de recidiva a largo plazo. Por último, la vigilancia estrecha en el seguimiento de pacientes identificados como individuos de riesgo para recidiva permite una detección oportuna con una disminución del tiempo de espera para la reintervención o cambios en el manejo y un mejor pronóstico. 


\section{BIBLIOGRAFÍA}

1. Toniato A, Boschin I, Casara D, Mazzarotto R, Rubello D, Pelizzo M. Papillary Thyroid Carcinoma: Factors Influencing Recurrence and Survival. Ann Surg Oncol 2008; 15: 1518-22.

2. Yip J, Orlov S, Ollov D, Vaisman A, Gomez Hk, Etarsky $D$, ET AL. Predictive value of metastatic cervical lymph node ratio in papillary thyroid carcinoma recurrence. Head Neck 2013; 35: 592-8.

3. Prescott JD, Sadow PM, Hodin RA, Phi Le L, Gaz RD, Randolph GW, et al. Braf v600e status adds incremental value to current risk classification systems in predicting papillary thyroid carcinoma recurrence. Surgery 2012, 152: 984-90.

4. Giovanella L, Trimboli P, Verburg FA, Treglia G, Piccardo A, Foppiani L. et al. Thyroglobulin levels and thyroglobulin doubling time independently predict a positive 18F-FDG PET/CT scan in patients with biochemical recurrence of differentiated thyroid carcinoma. Eur J Nucl Med Mol Imaging 2013; 40: 874-80.

5. Wong H, Wong Ke, Yau T, Tang V, Leung R, Chiu J, et AL. Is there a role for unstimulated thyroglobulin velocity in predictin recurrence in papillary thyroid carcinoma patients with detectable thyroiglobulin after radioiodine ablation? Ann Sur Oncol 2012; 19: 3479-85.

6. Yong JS, HyunguJ K, Su-Jin K, June YC, Kyu EL, Young JP, ET AL. Factors affecting the loco regional recurrence of conventional papillary thyroid carcinoma after surgery: A retrospective analysis of 3381 patients. Ann Surg Oncol 2015; 22: 3543-9.

7. GRANT CS. Recurrence of papillary thyroid cancer after optimized surgery. Gland Surgery 2015; 4: 52-62.

8. Trivizkı 0, Аmıt M, Flıss DM, GıL Z. Elective central compartement neck dissection in patients with papillary thyroid carcinoma recurrence. Laryngoscope 2013, 123: 1564-8.
9. Hassan A, Razi M, Riaz S, Khalid M, Nawaz MK, Syed AA, BAshir H. Survival analysis of papillary thyroid carcinoma in relation to stage and recurrence risk. Clin Nucl Med 2016; 41 : 606-13.

10. Chang WL, Jong-Lyel R, Gyungyup G, Kyung-Ja C, Seung-Ho C, Soon YN, et al. Risk factor for recurrence of papillary thyroid carcinoma with clinically node-positive lateral neck. Ann Surg Oncol 2015; 22: 117-24.

11. Chéreau N, Buffet C, Trésallet C, Tissier F, Leenhardt L, Menegaux F. Recurrence of papillary thyroid carcinoma with lateral cervical node metastases: Predictive factors and operative management. Surgery 2016; 159: 755-62.

12. Hurtado-López M, Melchor-Ruan J, Basurto-Kuba E, Montes De Oca-Duran Er, Pulido-Cejudo A, Athie-Gutierrez C. Recurrencia del cáncer papilar de tiroides con tiroidectomía total y terapia adyuvante 0 con cirugía limitada en grupos de bajo riesgo. Cir Cir 2011; 79: 118-25.

13. Ito Y, Miyauchi A, Kinara M, Fukushima M, Higashiyanna T, Miya A. Overall Survival of Papillary Thyroid Carcinoma Patients: A singleinstitution long-term follw-up of 5897 patients. World J Surg 2018; 42: 615-22.

14. Ito Y, Myauchi a; Kudo T, Kinara M, Fukushima M, MıYA A. The effectiveness of prophylactic modified neck dissection for reducing the development of lymph node recurrence of papillary thyroid carcinoma. World J Surg 2017; 41: 2283-9.

15. Haugen, Alexander. 2015 American Thyroid Association Management Guidelines for Adult Patients with Thyroid Nodules and Differentiated Thyroid Cancer: The American Thyroid Association Guidelines Task Force on Thyroid Nodules and Differentiated Thyroid Cancer. Thyroid 2016; 26: 1-133. 